\title{
Self-tonometry in glaucoma
}

\author{
Ahti Tarkkanen • Kristian Ulfves • Tuulikki Ulfves
}

Received: 21 March 2010/Revised: 9 May 2010/Accepted: 14 May 2010 /Published online: 8 June 2010

(C) The Author(s) 2010. This article is published with open access at Springerlink.com

\section{Dear Editor,}

Elevated intraocular pressure (IOP) is the main risk factor for glaucoma. It is also the only risk factor which can be modified by medical or surgical means. For its measurement, Goldmann applanation tonometry (GAT) is the golden standard. IOP may show marked variations over the course of the day and from day to day. While GAT is available only in hospitals and doctors' offices, with occasional measurements these relevant variations may be missed.

Experiences on home tonometry using the Schiötz tonometer were reported already in 1965 [3]. This, however, needs another person to perform the measurements and the use of local anesthetic drops, and there is a risk of corneal injury or impaired vision. Hence, the idea of self-tonometry was invented in 1967 [1]. In a recent exhaustive review, the present techniques for home and self-tonometry have been described [6].

The new Tiolat iCare tonometer was described in 1997 [4]. It is a hand-held unit which measures the deceleration of a magnetized probe in an electromagnetic field on the rebound from the cornea. It is also a non-contact unit, and no anesthetic drops are required. Its use as a self-tonometer has briefly been mentioned [5]. There is also a summary of the

\footnotetext{
A. Tarkkanen $(\bowtie)$

Helsinki University Eye Hospital,

Kapylantie 36,

00600 Helsinki, Finland

e-mail: ahti.tarkkanen@kolumbus.fi

K. Ulfves

Health Center of the City of Espoo,

Espoo, Finland

T. Ulfves

Department of Occupational Health, Diacor Ltd,

Espoo, Finland
}

literature comparing the iCare tonometer with GAT [2]. Whatever the IOP level, the results of iCare IOP measurements were well-correlated with GAT.

Our patient is a 44-year old female physician, who was seen by the senior author for the first time at the age of 13 years. There was a history of myopia since the age of 5 years. On examination, the visual acuity (VA) of the right eye (RE) was 0.8 with correction, the eye appeared externally quiet, IOP by GAT was $42 \mathrm{mmHg}$ and the optic disc showed temporal cupping. The VA of the left eye (LE) was 0.6 , IOP $50 \mathrm{mmHg}$ and the optic disc was also cupped. The anterior segment changes of both eyes were compatible with Axenfeld-Rieger type anomaly. The chamber angles were wide open, with strands of attached peripheral iris tissue. The patient has remained in the care of the senior author, and all means have been used to maintain the IOPs at low levels. Over the years, in addition to maximum medical therapy, a trabeculectomy and a transscleral krypton-laser cyclophotocoagulation have been performed to the RE. Glaucoma of the LE has required three filtering operations also combined with mitomycin, and a placement of an Ahmed Glaucoma Valve implant in 2008.

Home tonometry using the Schiötz tonometer was started by the patient in 2000. Her husband made the measurements of IOPs. However, the technique was felt to be clumsy, another person was always required; the anesthetic drops outdated rapidly, and were not always available in the pharmacy. Even cleansing of the instrument was felt to be troublesome.

In 2007, the patient made a trial with an iCare tonometer. Soon a decision was made to buy one for their home. In the beginning, the husband was asked to make the measurements. However, quite soon it was noted that self-tonometry by the patient gave a more accurate yield. Thereafter, only selftonometry has been practiced. The patient is measuring her 


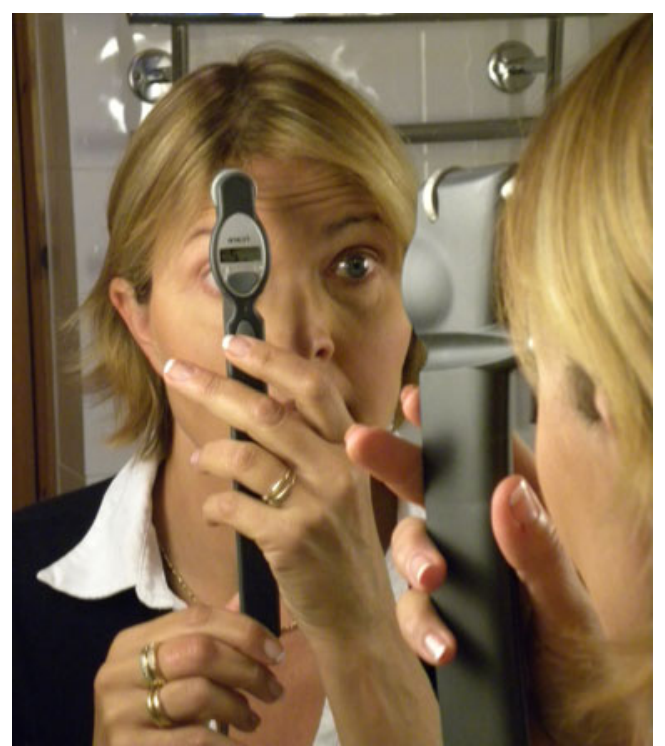

Fig. 1 Self-tonometry with an iCare unit. The patient in the right edge of the picture is measuring IOP from her LE through a mirror, while the RE is measured directly without an aid (with the patient's consent)

IOP from the RE directly without any aid, but the measurements of the LE are made using a mirror as an aid (Fig. 1). The patient's measurements have been checked against GAT values in the office, with very good agreement. Figure 2 shows an example of a 9-day daily tension curve, showing that glaucoma of the LE is way out of control. Using the iCare unit at home, chronic secondary open-angle was diagnosed also in the patient's 12-year-old son, with IOPs of 38 and $36 \mathrm{mmHg}$. For him, home tonometry is now practiced by the mother.

Features of the iCare tonometer considered negatively by the patient are the price of the instrument and the price of the removable tips of the probe, as well as the learning curve. No ocular complications have been encountered.
For the adequacy of glaucoma therapy measurements of IOP by GAT, study of the visual fields and the changes in the posterior segment are recorded. Unfortunately, the main risk factor, IOP, is too seldom measured. Particularly, the early morning spikes of IOP are missed. In one study using selftonometry, half of the IOP peaks occurred outside normal office hours, and elevated readings were recorded on patients with documented progression of glaucomatous damage [8].

In the practice of medicine today, patients play an active role in monitoring their diseases. Diabetics may alter their daily insulin dosing by frequent measurements of the blood glucose at home. For patients with arterial hypertension, a European recommendation has been issued for ambulatory and home blood-pressure measurements [7].

One would hope that self-tonometry could make an important contribution of glaucoma therapy in the future, at least in selected cases. A suitable level of responsibility and motivation are required by the patient. For patients unable to adopt self-tonometry, home tonometry by a family member might be suggested. In addition to iCare, there are other units available for self tonometry, e.g. Ocuton [6], which provides suitable records from diurnal IOP home tonometry. The results could be send to a central database, at least in remote areas. A plea is made to the manufacturers that there will be improvements in the technique. Future self-tonometry units should be reliable, non-contact units, easy to learn and maintain. In the case of high purchase prices, the possibility of a loan from the health center, hospital or doctor's office should be considered. At least in the case described, self-tonometry has helped in finding large variations of IOP leading to immediate surgical procedures and the preservation of vision.

Notice: The authors have no proprietory interest in the iCare tonometer. The research plan was approved by the Committee of Medical Ethics.
Fig. 2 Self-tonometry with an iCare unit. A 9-day daily tension curve reveals that the IOP of the LE is out of control on maximum medical therapy, with variations between 18 to $37 \mathrm{mmHg}$. Early measurements were performed at 7 a.m. and the late ones at midnight

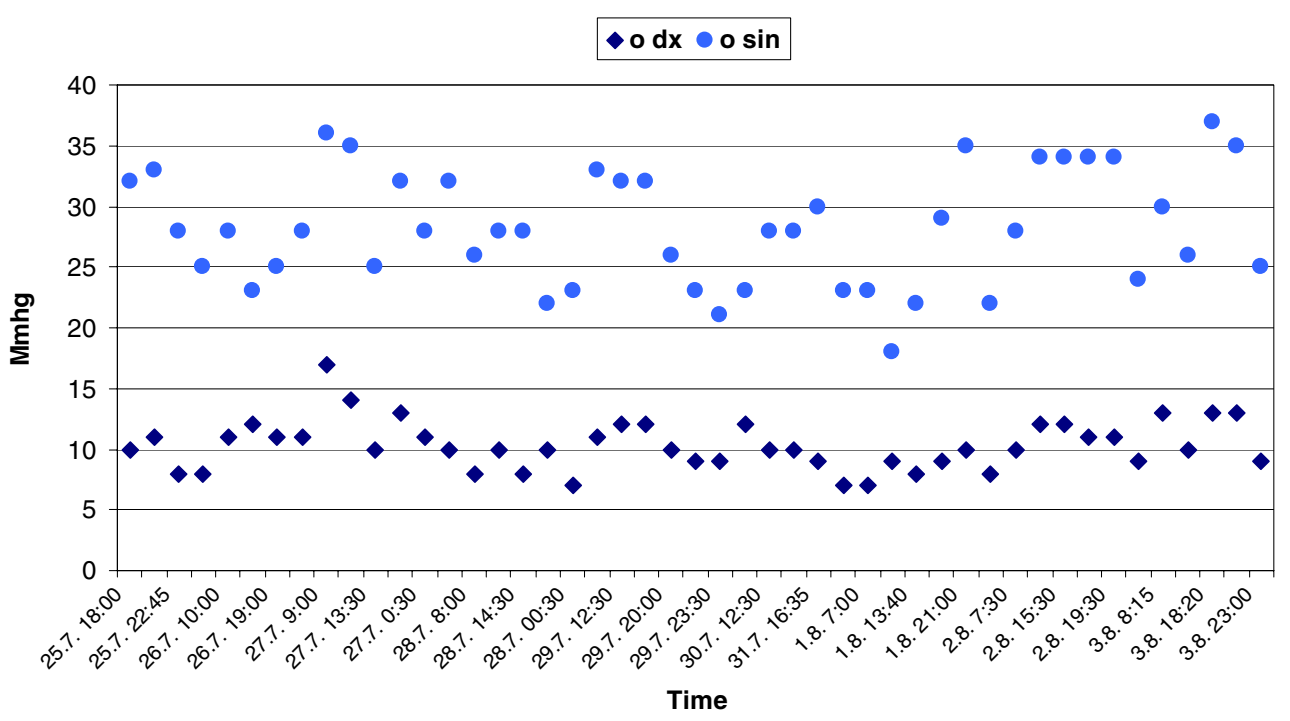


Open Access This article is distributed under the terms of the Creative Commons Attribution Noncommercial License which permits any noncommercial use, distribution, and reproduction in any medium, provided the original author(s) and source are credited.

\section{References}

1. Collins CC (1997) Miniature passive pressure transensor for implanting in the eye. IEEE Trans Biomed Eng 14(2):74-83

2. Detry-Morel M (2007) Update in rebound tonometry. Phosphene and rebound tonometries, self-tonometry and technologies for the future. Bull Soc Belge Ophtalmol 303:87-95

3. Jensen AD, Maumenee AE (1965) Home tonometry. Amer J Ophthalmol 76:929-932
4. Kontiola A (1997) A new induction-based impact method for measuring intraocular pressure. Doc Ophthalmol 93(3):265-276

5. Kontiola A, Puska P (2004) Measuring intraocular pressure with the Pulsair 3000 and Rebound tonometers in elderly patients without an anesthetic. Graefes Arch Clin Exp Ophthalmol 242(1):3-7

6. Liang SY-W, Lee GA, Shields D (2009) Self-tonometry in glaucoma management - past, present and future. Surv Ophthalmol 54:450-462

7. O’Brien E, Asmar R, Beilin L, Imai Y, Mallion J-M, Mancia G, Mengden T, Myers M, Padfield P, Palatini P, Parati G, Pickering T, Redon J, Staessen J, Stergiou G, Verdecchia P (2003) European society of hypertension recommendations for conventional, ambulatory and home blood pressure measurement. J Hypertens 21:821-848 8. Wilensky JT, Gieser DK, Mori MT, Langenberg PW, Zeimer RC (1987) Self-tonometry to manage patients with glaucoma and apparently controlled intraocular pressure. Arch Ophthalmol 105:1072-1075 